\title{
Rotor Shape Multi-Level Design Optimization for Double- Stator Permanent Magnet Synchronous Motors
}

\author{
Pedram Asef, Member, IEEE, Ramon Bargallo Perpina, Saeed Moazami, Andrew C. Lapthorn, Member, IEEE,
}

\begin{abstract}
This research presents a rotor shape multi-levelobjective optimization designed to reduce the mechanical stress distribution in the rotor core of a double-stator permanent magnet synchronous motor. The second objective is weight minimization performed via a response surface methodology (RSM) with a uniform precision central composite design (UPCCD) function. The optimal operation point, with a substantial population size, is reached using a Monte Carlo algorithm on the fitted model. The goodness-of-fit for the model is evaluated based on the modified Akaike information criterion (AICc) and the Bayesian information criterion (BIC) with a linear regression approach. To achieve these goals, a multi-level design procedure is proposed for the first time in machine design engineering. All the electromagnetic forces of the machine such as normal, tangential, and centrifugal forces are calculated using 3-D transient finite element analysis (FEA). The outcome of the proposed rotor core optimization shows that the finalized shape of the studied core has significantly smaller weight and mechanical stress, while the electromagnetic performance of the machine has remained consistent with a pre-optimized machine.
\end{abstract}

Index Terms--Finite Element Analysis, Multi-level Optimization, Monte Carlo Algorithm, Response Surface Methodology, Synchronous Machine, Stress Computation.

\section{INTRODUCTION}

$\mathrm{N}$ OWADAYS the pace of research is very coherent among researchers of double-stator permanent magnet brushless synchronous (DS-PMBL) machines to enrich the machine's performance [1-4]. This research is due to the high capability of this type of topology for variety of applications such as electric vehicles (EVs) and wind power generation. There are several challenging design issues, which must be carefully considered to avoid any faults. DS-PMBL topology, with two airgaps and stator cores, bring additional complexity to the electromagnetic and mechanical forces calculations that are the underlying information towards having a clean image of the stress distribution computation on the model, predominantly for high-speed applications. Mechanical stress research is readily found in the literature, as follow:

In reference [5], the authors presented the impact of mechanical stress on characteristics of interior permanent

Pedram Asef, and Professor Ramon Bargallo Perpina are with the Department of Electrical Engineering, Technical University of Catalonia-BarcelonaTech, EEBE, 08019, Barcelona, Spain

(e-mail: pedram.asef@upc.edu; ramon.bargallo@upc.edu).

Saeed Moazami is with Lamar University, Electrical Engineering Department, Beaumont, Texas, USA, (email: smoazami@lamar.edu).

Dr. Andrew Lapthorn is a Senior Lecturer for the Electrical and Computer Engineering Department, University Of Canterbury, Christchurch, New Zealand (email: andrew.lapthorn@canterbury.ac.nz). magnet synchronous motors from both results of measurement and calculation. As outcome of the study, they clarified that the mechanical stress causes not only an increase in the stator iron loss, but also an increase in the rotor-iron loss, and a decrease in the reluctance torque of interior permanent magnet synchronous motors. In another work, the researchers studied the correlation between rotor vibration and mechanical stress in ultra-high-speed permanent magnet synchronous motors (PMSMs); the mechanical stress generated in the PM and the rotational vibration when the PMSM rotates at high speeds were evaluated [6]. They have presented the stress variation as function of shrink fit in a variable speed analysis.

$\mathrm{X}$. Sun et al. [7] worked on the accurate suspension force model of a Bearingless PMSM different from conventional suspension force models, a modeling scheme for the suspension force of a Bearingless PMSM is presented by considering rotor eccentricity with the Maxwell stress tensor modeling scheme.

F. Chai et al. [8] proposed a novel analytical method to calculate the maximum mechanical stress (MMS) on the rotor of the interior permanent-magnet synchronous motor with $\mathrm{V}$ shaped rotor structure. They have realized that an appropriate $\mathrm{V}$-shaped angle and the central bridge thickness are needed through the theoretical calculation. The number of pole pairs is reduced by adjusting the slot/ pole combination to enhance the electromagnetic performance. Finally, the geometric parameters of the PMs slightly change, to minimize MMS.

Although, the mentioned studies to compute stress are significantly advancing analytical, numerical, and experimental investigations in this area. But, there are mostly dealing with simple machine topologies such as SPM and IPM. A lack of research in stress and deformation computations for DS-PMBL topology (which has a complex topology) has drawn the attention of this study. Some recent research regarding the force calculation problem is summarized below, where the researchers have faced the electromagnetic-mechanical calculations for complex machine topologies.

A. H. Isfahani and B. Fahimi [9] studied the mechanical vibration analysis of a double-stator switched reluctance machine (DSSRM). As findings of the research, they have proposed DSSRMs because of radial force reductions in comparison with conventional switched reluctance machines (SRMs). The vibration and acoustic noise analysis were considerably decreased as a result.

In another study, the researchers have proposed a new flattype cup-rotor DDRM, where they have reduced the radial force, which has increased the efficiency. The paper's achievement has been energy efficiency maximization, which 
has been analytically, numerically, and experimentally verified [10]. These two-notable works [9-10] have presented how the electromagnetic forces resulted in mechanical vibrations in a complex machine topology.

W. Wang et al. [11] investigated the optimal rotor shape design of a DSSRM. They have analyzed the impact of shape on the torque profile and afterwards developed an iterative method, which automatically shapes the rotor using static finite element analysis (FEA).

Based on the addressed literatures, the importance of sizing optimization which affects the forces plays an important role. Additionally, an accurate electromagnetic force computation is required prior to any mechanical-based analysis.

In this study, the fundamental electromagnetic-mechanical forces such as radial, tangential, and centrifugal that are present in the stress distribution of a DS-PMBL have been studied. Mechanical stress computation using 3-D transient FEA on the rotor core is investigated in which a response surface method with central composite design (CCD) functions were used to reduce the mechanical stress and weight based on a sizing optimization while the power density is unchanged. Unlike a conventional analysis of variance, which is used for simple experiments where parameters are randomized and replicated, Bayesian information criterion (BIC) provides more accuracy by accounting for mixed effects and is nested, in which a linear regression model is needed. To summarize the contribution of the study:

- Fundamental electromagnetic and mechanical forces calculation of a DS-PMBL using a 3-D FE model (transient condition)

- Mechanical stress transient analysis of in-set permanent magnet (PM) rotor core

-Defining three controllable variables, in which the volume of the permanent magnet is fixed.

-Evaluating findings through AICc and BIC and regression model

- Searching for the best possible optimal operation point at the first-level through the response surface method (RSM), where all objectives and optimization constraints are satisfied.

- Generating $n$ dimension samples around the optimal point (first level) to obtain the second-level optimum point using the Monte Carlo algorithm.

\section{FORCES CALCULATION OF DS-PMBL}

Due to high permeability of the material used in the stator and rotor cores, in contrast with the air permeability, the force density in the steel lamination materials of the DS-PMBL is ignored. The mechanical properties of the materials of the rotor core with in-set permanent magnets is shown in Table I.

Fig. 1 illustrates a comprehensive 3-D view of the structure of a DS-PMBL, in which the three-phase machine's winding configuration in both stator cores are shown in Fig. 1(a). The electromagnetic capability of the winding configuration has been completely studied and verified in [1]. Fig. 1(b) presents
TABLE I. Mechanical Properties OF THE Rotor MATERIAL

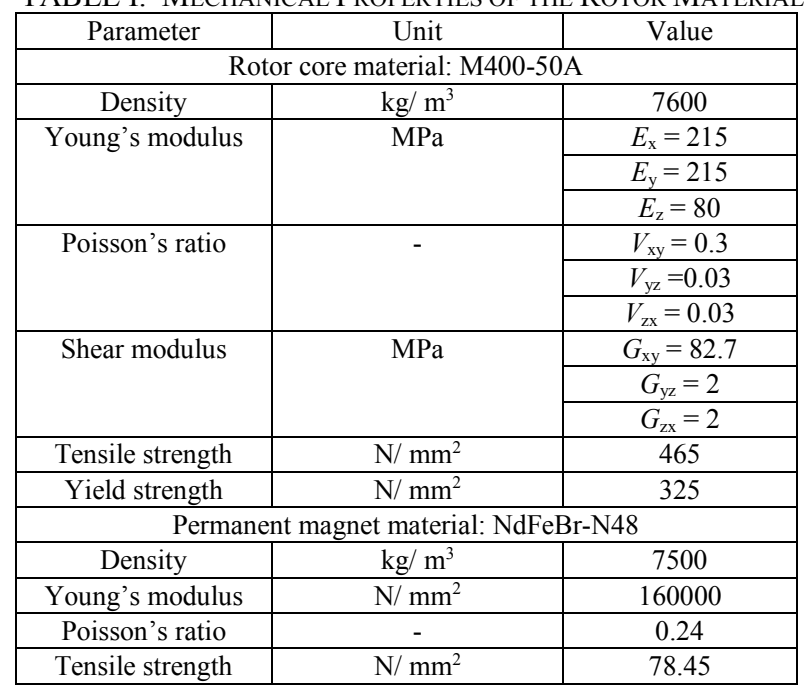

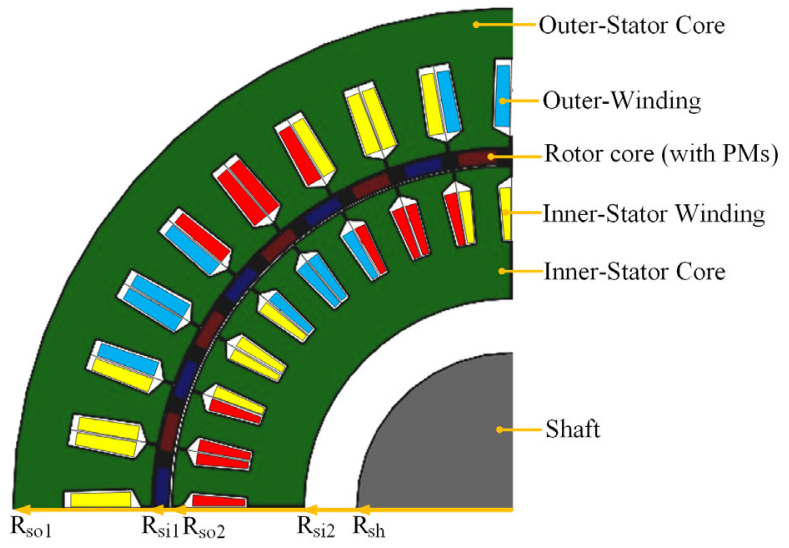

(a)

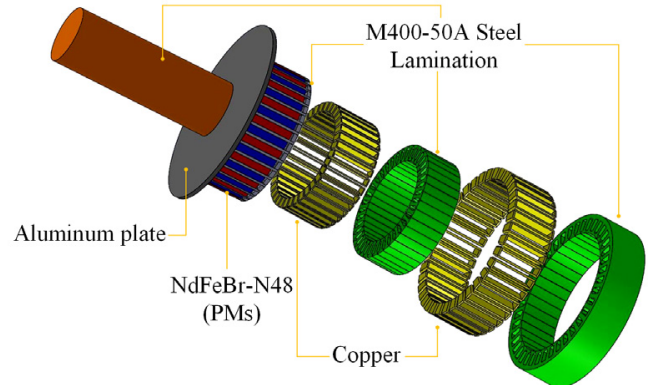

(b)

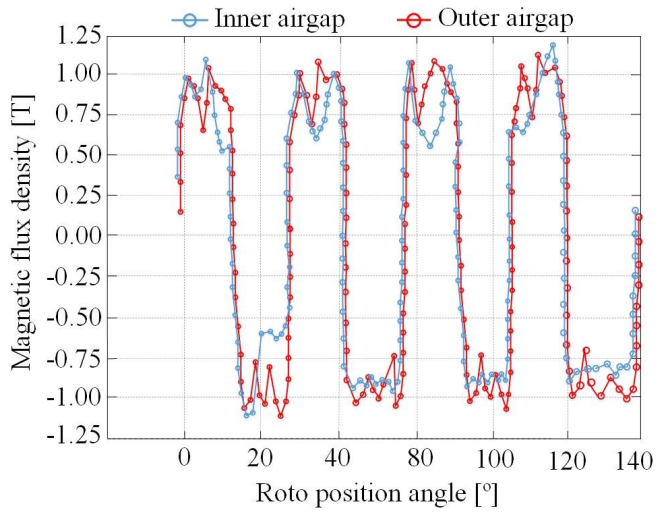

(c)

Fig. 1. Physical structure view of the DS-PMBL, (a) winding configuration, (b) extruded view of completed design of the machine and (c) the inner and outer airgap flux density as a function of rotor position. 
3-D physical structure of the individual major parts of the machine in an exploded view such as both stators and their coils, the rotor core and its PMs, as well as the shaft. The PMassist segmented rotor core is fastened to the shaft using an aluminum plate with multi-directional locking screws to enhance the angular stability and resistance to fatigue, stress, and cracking. In this unique structure, the shaft is placed on one bearing, which decreases the machine cost and friction. Also, the rotor structure is designed to be connected directly to the load as direct-drive without the shaft requirement. Therefore, the aluminum plate is directly connected to the load utilizing only one bearing and without the use of a shaft. This unique homogeneous structure allows the rotor to operate in high-speed, high-temperature, harsh environments, as well as servo applications. More details can be found in [10], where it is validated. Fig. 1(c) indicates the inner and outer magnetic flux density waveforms of the studied machine. The stator and rotor core are made with M400-50A non-orientated electrical steel, and the PM material is NdFeBr-N48 (Table I).

The electromagnetic forces calculation methodology of a DS-PMBL is investigated based on 3-D FEA. A force vectors schematic is presented in Fig. 2, where all of the studied components, such as normal $\left(F_{\mathrm{N}}\right)$, tangential $\left(F_{\mathrm{T}}\right)$, and centrifugal $\left(F_{\mathrm{C}}\right)$ forces are considered. The green dashed line shows the path of magnetic flux density through the rotor core between windings of both stators. Both electromagnetic-based components, normal and tangential forces $\left(B_{\mathrm{n}}, B_{\mathrm{t}}\right)$ are calculated using the Maxwell stress tensor equation [11-12] and the electromagnetic torque of the machine, respectively. Likewise, the centrifugal force is purely computed using the transient 3-D FEA (via Abaqus software) at the nominal rotational speed. The torque density production of the machine is independent from $F_{\mathrm{N}}$ and $F_{\mathrm{C}}$; therefore, the torque density can be estimated directly from $F_{\mathrm{T}}$. Each of the force components is crucial for the deformation and mechanical stress issues regarding the machine's application. These force components are highly dependent on the electromagnetic behavior (forces distribution) and material used of the machine. For example, $F_{\mathrm{C}}$ increases while the rotor is rotating with higher angular velocity.

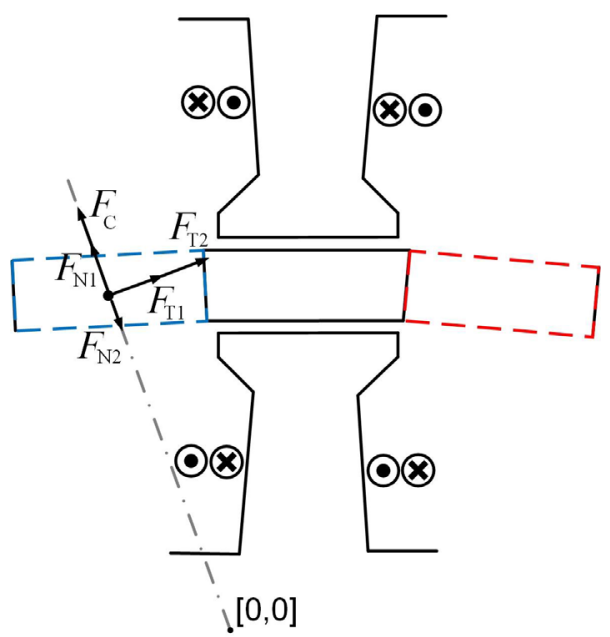

Fig. 2. Demonstration of normal $\left(F_{\mathrm{N} 1}-F_{\mathrm{N} 2}\right)$, tangential $\left(F_{\mathrm{T} 1}+F_{\mathrm{T} 2}\right)$, and centrifugal $\left(F_{\mathrm{c}}\right)$ force components in a two-dimensional DS-PMBL.

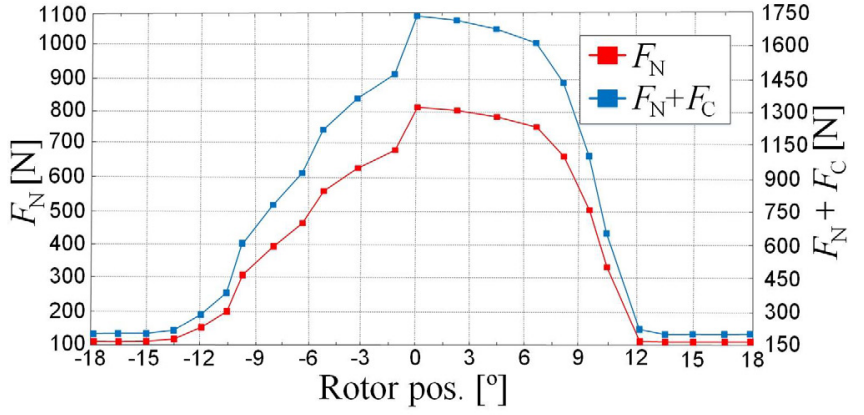

Fig. 3. On-load maximum radial force (normal and centrifugal components) of the DS-PMBL under $3 \mathrm{~A}$ excitation current with $30^{\circ}$ rotation.

The components of force can be computed [10] through the following equations:

$$
\begin{aligned}
& F_{N}=\sum F_{N 1}+F_{N 2}=\frac{1}{2 \mu_{0}}\left(\left(B_{n 1}^{2}-B_{t 1}^{2}\right)-\left(B_{n 2}^{2}-B_{t 2}^{2}\right)\right) \\
& F_{T}=\sum F_{T 1}+F_{T 2}=\frac{1}{2 \mu_{0}}\left(\left(B_{n 1}^{2} \times B_{t 1}^{2}\right)+\left(B_{n 2}^{2} \times B_{t 2}^{2}\right)\right) \\
& F_{C}=m \omega^{2} r
\end{aligned}
$$

where $\mu_{0}$ is the absolute permeability, and $B_{\mathrm{n}}, B_{\mathrm{t}}$ are normal and tangential magnetic flux density. $m$ is the mass of rotational part(rotor), $\omega$ is the rotational speed, and $r$ is the radius of the rotor.

Fig. 3 shows the produced normal forces $\left(F_{\mathrm{N} 1}, F_{\mathrm{N} 2}\right)$ from the outer and inner excited coils have opposite vector directions, which provides a vector as $F_{\mathrm{N}}=F_{\mathrm{N} 1}-F_{\mathrm{N} 2}$ (red curve as shown in Fig. 3). Fourier analysis is employed to compute amplitude and phase of each spatial harmonic order of the flux density distribution in the air-gap, where load condition for the analysis of magnetic forces by transient electromagnetic simulations was considered. Both the normal and centrifugal forces have a distribution with a radial vector direction have an overlapped influence on each other, where a summation of them, leading up to the resultant blue curve in Fig. 3, which indicates the total radial force on the rotor. The quantity of is only added to $F_{\mathrm{N}}$ (which changes at each angle), and thus $F_{\mathrm{C}}$ itself is not a function of rotor angle.

The normal magnetic force density in the air-gap is defined as follow:

$$
F_{n}(\theta, t)=F_{n, m} \cos (m \theta-k \omega t)
$$

where $\theta$ and $t$ are the angular mechanical position and time, $k$ denotes the time harmonic order, and $\omega$ is angular velocity. $m$ index shows the mode number.

Fig. 4 illustrates the rated produced electromagnetic torque (T) of the studied DS-PMBL, which is directly proportional with $F_{\mathrm{T}}$. Under 15 A peak current, maximum torque of 119 N.m is reachable. When the rotor (with PMs-assist) pass one unaligned position $\left(-18^{\circ}\right.$ or $\left.18^{\circ}\right)$ toward the next unaligned position by a $36^{\circ}$ rotation while the armature current is $25 \mathrm{~A}$ which offers a maximum torque of $145 \mathrm{~N} . \mathrm{m}$. The effective $F_{\mathrm{N}}$ has increased with a nonlinear gradient. As depicted, the maximum torque is achievable at the aligned position of -6 to -9. Under over load condition, a larger current (i.e. 40 A) increases the maximum torque up to $168 \mathrm{Nm}$. It should be mentioned that the cores of the machine will be saturated while the machine runs over rated speed (1000 rpm) and 


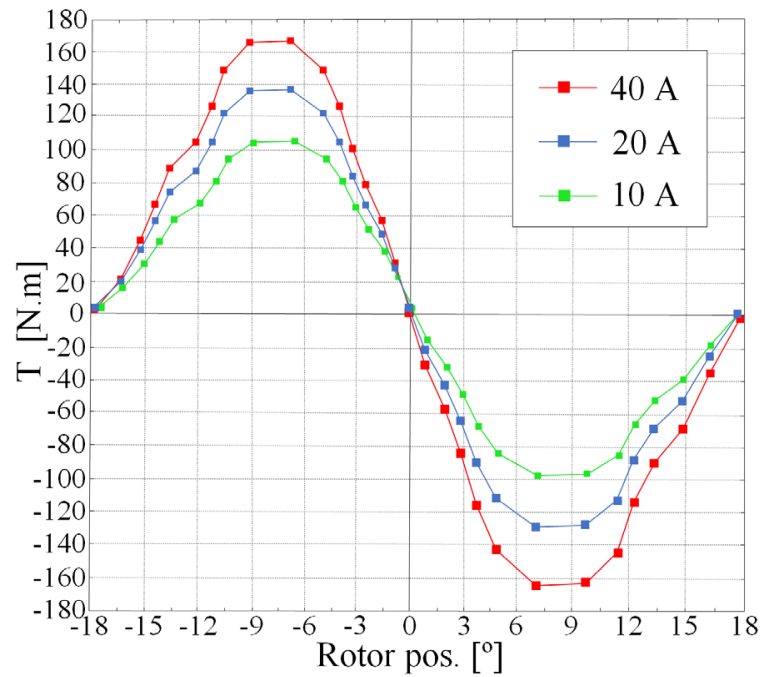

Fig. 4. Nominal electromagnetic torque of the studied DS-PMBL.

consequently the resulted current of over $20 \mathrm{~A}$ is produced.

Based on the calculated force components of the machine, the next section presents a transient 3-D FEA simulation setup to predict the mechanical stress distribution and deformation of the rotor as output of the study. Afterwards, three main geometrical design factors of the rotor were found through an effect test, where those design variables are employed for a multi-objective optimization using the response surface methodology in the optimization section. The optimization goals are minimization of the mechanical stress and weight of the DS-PMBL's rotor core.

\section{Model FitTing AND PRoBlem Definition}

The mechanical strength of the rotor relies on a number of parameters such as thermal stress, rotor vibration and deformation, as well as transient operation, which consists of the unsteady behavior of the machine. The theory of mechanical transient analysis can be addressed in [15]. The following assumptions and conditions are applied to the simulation setup (3-D transient FEA) when the rotor mechanical strength of the DS-PMBL was simulated at the nominal rated speed of $1000 \mathrm{rpm}$.

1) Mathematical modeling of the 3-D structure of the rotor with hexahedron mesh type (eight-node linear brick) and 71382 elements.

2) An advanced type of transient solver known as dynamic, explicit is performed to increase the accuracy.

3 ) The rotor material properties are adopted for the nonoriented lamination electrical steel M400-50A and $\mathrm{NdFeBr}-\mathrm{N} 48$ (details are presented in Table I).

4) Thermal effects and vibration are neglected.

5) Rotor eccentricity is considered via the simulation setup.

The DS-PMBL is a fractional-slot concentrated winding (FSCW) type with 36 slots in each stator core and $40 \mathrm{PMs}$ in the rotor. The stator is a segmented-teeth-type for a closed-slot topology. The rotor has its PMs embedded by means of injected molding, which provides a hybrid torque generation. More details can be found in [6].
Fig. 5 illustrates the sensitivity analysis over a number of design variables, where the variables with highest absolute contrast are considered. Also, t-ratio and individual p-values are calculated to provide the best selection process for the variables. The selected variables have highest t-ratio and approximately zero p-values. These geometrical-based controllable variables of the rotor core are listed as $x_{1}$, through $x_{5}$ which are; the width of axial rotor core tangency with the PMs, the radial width of the rotor core, the rotor bezel, the length of the air-gaps on both-sides and the angle-cut of the rotor core, respectively. As shown in Fig. 5, $x_{1}$ with a t-ratio of 4.997 and a p-value of 0.0187 has the most effect on the mechanical stress $\left(y_{1}\right)$ and weight of the rotor core $\left(y_{2}\right)$ objectives, while $x_{2}$ and $x_{3}$ are the next most significant factors with p-values of 0.0378 and 0.0792 respectively. Even though the maximum stress is lower than yield strength of core material, the rotor core could be damaged as time passes because of the fatigue characteristics of material (M400-50A). A high-cycle fatigue model is considered.

TABLE II. DESIGN VARIABLES OF THE ROTOR CORE

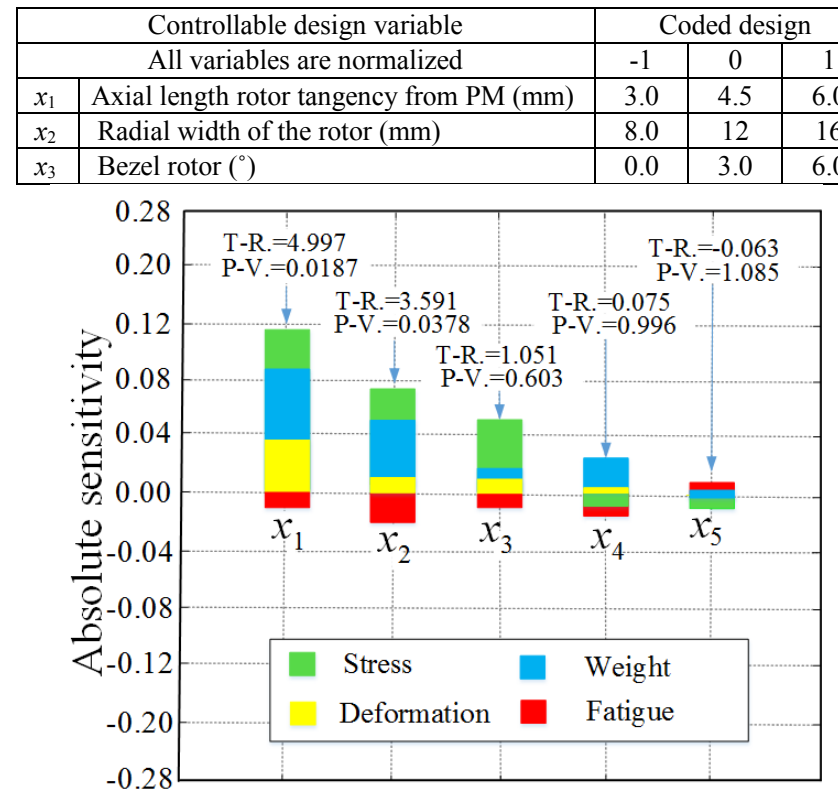

Fig. 5. Sensitivity analysis of the highest absolute contrast design factors.

Thus, the maximum stress of the rotor core has to be lower than the fatigue limitation. The typical fatigue limit for the rotor is based on $0.5^{*}$ ultimate tensile strength (which is 445MPs for M400-50A) [21]. Furthermore, the maximum displacement of the prototype should be considered against clearance limit after the stress computation.

The second-order polynomial model for the stress as the first objective $\left(y_{1}\right)$ is given as:

$$
\begin{aligned}
& y_{1}=\beta_{0}+\sum_{i=1}^{k} \beta_{i} x_{i}+\sum_{i=1}^{k} \beta_{i i} x_{i}^{2}+\sum_{i<j} \sum \beta_{i j} x_{i} x_{j}+\epsilon_{i j}= \\
& 71.502719-2.092663 \mathrm{X}_{1}-1.237483 \mathrm{X}_{2}- \\
& 3.38254470523556 \mathrm{X}_{3}+0.279560\left(\mathrm{X}_{1} \cdot \mathrm{X}_{2}\right)+1.285714\left(\mathrm{X}_{1} \cdot \mathrm{X}_{3}\right) \\
& +0.180128\left(\mathrm{X}_{2} \cdot \mathrm{X}_{3}\right)-0.139849380630383\left(\mathrm{X}_{1} \cdot \mathrm{X}_{1}\right)- \\
& 0.032689\left(\mathrm{X}_{2} \cdot \mathrm{X}_{2}\right)-0.24719711427643\left(\mathrm{X}_{3} \cdot \mathrm{X}_{3}\right)+0.05
\end{aligned}
$$

and for the second objective $\left(y_{2}\right)$, the weight of rotor core is: 


$$
\begin{aligned}
& y_{2}=1.358580+0.050381 \mathrm{X}_{1}+0.049113 \mathrm{X}_{2}+0.002814 \mathrm{X}_{3} \\
& -0.015065\left(\mathrm{X}_{1} \cdot \mathrm{X}_{2}\right)-0.0035\left(\mathrm{X}_{1} \cdot \mathrm{X}_{3}\right)-0.000967\left(\mathrm{X}_{2} \cdot \mathrm{X}_{3}\right) \\
& +0.006095\left(\mathrm{X}_{1} \cdot \mathrm{X}_{1}\right)+0.005173\left(\mathrm{X}_{2} \cdot \mathrm{X}_{2}\right)-0.001912\left(\mathrm{X}_{3} \cdot \mathrm{X}_{3}\right)+0.005
\end{aligned}
$$

where $x$ is a $(k \times 1)$ vector of the treatments. $\beta$ is a $(k \times k)$ symmetrical matrix that consists of pure quadratic coefficients $\left(\beta_{i i}\right)$ in the main diagonal elements. For off-diagonal elements, one-half of the mixed quadratic coefficients $\beta_{i j}, i \neq j . \varepsilon$ is the error observed in the response of the firstlevel $y_{1} . \varepsilon_{\mathrm{ij}}$ integrates any other sources of variability in the experiment, which consists of measurement variability arising from noise and differences among units [16].

Regarding the above polynomials, the following desirability function has been applied:

$$
\begin{aligned}
& D=\left[w_{1} \operatorname{Ln}\left(d_{1}\right)+w_{2} \operatorname{Ln}\left(d_{2}\right)\right], \text { where }: 0 \leq w_{i} \leq 1 \\
& f(x)_{i}=e^{0.5}\left(\operatorname{Ln}\left(d\left([30,55,80],[0.9819,0.5,0.066], y_{1}\right)\right)\right. \\
& \left.+0.5 \operatorname{Ln}\left(d\left([1,2.5,4],[0.9819,0.5,0.066] . y_{2}\right)\right)\right)
\end{aligned}
$$

where $w_{\mathrm{i}}$ is weight or importance level of $i$ th response as $w_{\mathrm{i}}$. Multi-objective optimization requires a multi-response desirability based on the geometric mean of the transformed responses $\left(d_{1}\right.$ and $\left.d_{2}\right)$.

\section{Model SELECTION AND Optimization Process of The STUDIED PROBLEM}

The procedure from the problem diagnosis to the multilevel optimization stage is proposed here for the first time in the machine design engineering. Fig. 6 presents how, toe-bytoe, the objectives are reached using the proposed procedure. First, the best variables are found through a uniform central composite design (UP-CCD) matrix consisting of 20 design of experiments beaning computed to fulfill the matrix. Then, a fitting model and model selection process have been applied to obtain the most suitable fitted model, which has the smaller error and is free from the over fitting problem. The chosen model has been optimized using a second order polynomial based on regression. Also, noise variables are defined for both objectives in the desirability function (Eq. 4). When the optimal operation point has been selected, the Monte Carlo algorithm is executed over 5000 iterations around the optimal point to determine a more optimal point via the training and test data.

\section{A. Fitting Model and Selection Process}

The most desirable model should be selected based on model fitting through the linear and second order polynomial approaches. The two advanced model selection approaches known as corrected the Akaike information criterion (AICc) [17] and the Bayesian information criterion (BIC) [18-19] methods, where the mathematical terms of the selection procedure are given as:

$$
\begin{gathered}
A I C c=2 k-2 \ln (\widehat{L})+\left(\frac{2 k^{2}+2 k}{n-k-1}\right) \\
B I C=\ln (n) k-2 \ln (\widehat{L})
\end{gathered}
$$

where $k$ is the number of estimated parameters in the model, $\hat{\boldsymbol{L}}$ is the maximum value of the likelihood function for the model and $n$ is the number of observations (sample size).
Fig. 7 presents the variation of $\triangle \mathrm{AICc}$ (blue) and $\triangle \mathrm{BIC}$ (red) values over different models sizes. For instance, $\triangle \mathrm{AICc}$ is the different between smallest AICc to the smallest overall. The smallest AICc model is the best in theory, but the AICc estimation values have considerable uncertainty, hence, $\triangle \mathrm{AICc}$ should be used. The best-fitted models are the 8,7 , and 6-terms with the smallest delta values. The averaging technique helps to determine the best agreement (or tradeoff), where the 6-terms model is proposed due to avoid any over fit issues despite the 8 terms model having the ideal smallest value (zero). Table III illustrates the summary of the fitted 6 terms model of the two objectives (stress and weight) based on a stepwise approach for the design variables used. A tradeoff between error level and the number of parameters is needed for each model, 6, 7, and 8 terms with the lowest value of AICc and BIC (results a better model fitting). The degree of freedom (DF) of the total error for both objectives $\mathrm{Y}_{1}$ and $\mathrm{Y}_{2}$ is 10 (including lack of fit and pure error distribution). The sum square error (SSE) of the models is presented and evaluated via root average square error (RASE) and Press-root mean square error factors. RASE is a standard deviation of the differences (residuals) between the predicted values and observed values when the calculations are performed over the data sample that was used for estimation and are called prediction errors. The smaller RASE and P-RMSE values are is more desirable because of the smaller prediction error among different models. An adjusted $\mathrm{R}^{2}$ tests the fit accuracy of the model for each individual objective, which was $97 \%$ and $98 \%$ for $y_{1}$ and $y_{2}$, respectively. The lack-of-fit errors are ignorable toward the large number of SSE, in addition, the adjusted $\mathrm{R}^{2}$ assessments.

\section{B. Setup of Multi-Objective Optimization Algorithm}

This study deals with a multi-level-objective design optimization (Based on Fig. 6) as its main contribution to minimize both mechanical stress and weight with weights of $w_{1}=1$ and $w_{2}=0.5$ (Eq. 4). As the weight reduction may cause a worse deformation $F(\mathrm{i})$, this parameter is considered as one of the optimization constraints. This research is focused only on the rotor core, where the finest type of modeling and meshing is carefully considered to reduce the volume of the $3-\mathrm{D} \mathrm{FE}$ model and processing time of the simulation. The FE simulations have been completed using an Intel ${ }^{\circledR}$ Xenon ${ }^{\circledR} \mathrm{CPU}$ E5530@2.40GHz with 12 GB of RAM, and a 64-bit operating system. 71382 hexahedron type mesh elements (the most suitable kind for 3-D modeling) were generated using 8node linear brick, reduced integration and hourglass control, which is shown in Fig. 8.

A tie constraint is utilized in Abaqus to model the contact between PMs and the rotor. In this method, each two corresponding surfaces are fused together and therefore there will be no relative velocity between related surfaces during simulation time. In fact, this model is used to bond two regions with dissimilar material properties or even different mesh types. After the matrix of UP-CCD has been filled with the design of experiments, the first-level of optimization using RSM is completed. The solver functions of standard least squares are applied to determine the first-level optimum point. Fig. 9 illustrates the response surface results from both objectives $\left(y_{1}\right.$ and $\left.y_{2}\right)$, and their individual relationships with 
the design variables $x_{1}, x_{2}, x_{3}$. Fig. 9(a) presents the variation of first objective (mechanical stress) for a range set of both $x_{1}$ and $x_{2}$. A smaller $x_{1}$ and larger $x_{2}$ results the minimum stress, which is the ideal target. Additionally, a similar observation was studied for variables $x_{2}$ and $x_{3}$ in Fig. 9(b). However, both

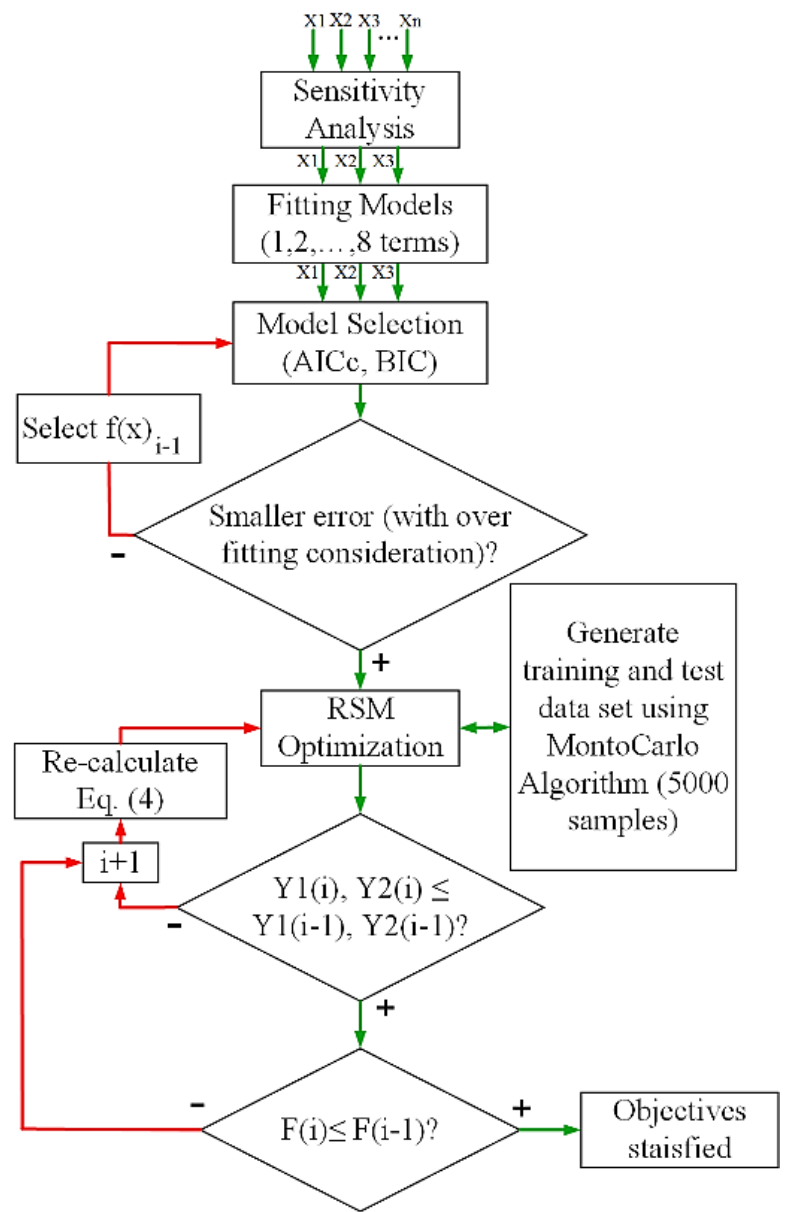

Fig. 6. Model selection and multi-level optimization flowchart.

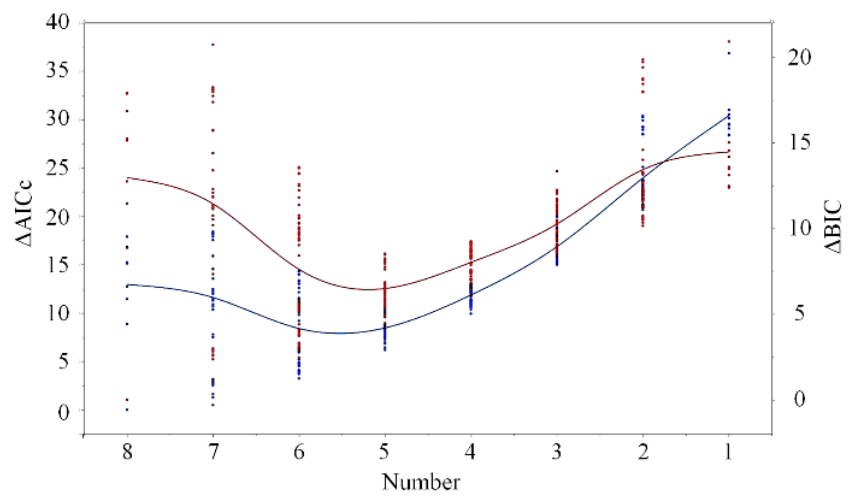

Fig. 7. Delta AICc and BIC demonstration over a range of models.

TABLE III. Summary Fit of 6-Terms Model Based on AICC AND BIC APPROACHES

\begin{tabular}{|c|c|c|c|c|c|c|c|}
\hline Source, $y_{1}$ & DF & SSE & RMSE & $\mathrm{R}^{2}$ A. & P-RMSE & AICc & BIC \\
\hline Model & 9 & 2561.9 & 11.611 & 0.975 & 2.9837 & 157.5 & 159.8 \\
\hline Error & 10 & 155.67 & - & - & - & - & - \\
\hline Lack of fit & 5 & 168.99 & - & - & - & - & - \\
\hline Source, $y_{2}$ & \multicolumn{7}{|c|}{ Note: $y_{2}$ as second objective which is weight. } \\
\hline Model & 9 & 3.4148 & 0.4239 & 0.982 & 2.1102 & 26.11 & 24.39 \\
\hline Error & 10 & 0.0899 & - & - & - & - & - \\
\hline Lack of fit & 5 & 0.0899 & - & - & - & - & - \\
\hline
\end{tabular}

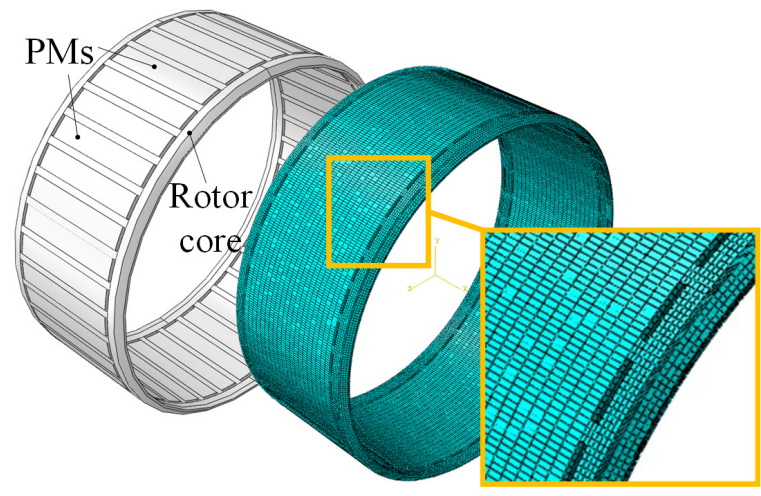

Fig. 8. 3-D geometry and its generated Hexahedron mesh.

variables tend to be considered smaller in order to minimize the mechanical stress. For the second objective (weight), the smaller $x_{1}$ and $x_{2}$ are predicted to produce weight reduction as shown in Fig. 9(c). $x_{2}$ is the most significant design factor to reduce the weight which is also shown in Fig. 9(d). This figure depicts the $x_{3}$ has little effect on the weight objective and also shows that $x_{2}$ tends to be smaller for achieving the goal of weight optimization. Additionally, the optimum point is predicted to be very close to the offset point line of the graphs.

The multi-level optimization concept begins from the idea of optimizing the optimal operation point from the RSM by 2.03, 2.98, and 0.17. Afterwards, the second-level drives a Monte Carlo algorithm using a training and test data set, where 5000 test samples are analyzed to determine the most optimal point around $(2.03,2.98,0.17)$.

Fig. 10 presents the training and test data for 5000 data points (samples), in which the black line illustrates the class prediction concept using the Monte Carlo algorithm with a linear regression solver. The test and training set have similar global features since they have been generated via the same random variables. The reason for creating test and training sets is to detect over-training through evaluation on different data than that used to fit the models or algorithms. The estimation probability of 1 is via a linear regression model with all the design variables $\left(x_{1}, x_{2}, x_{3}\right)$ as predictors. The 3-D prediction map (Fig. 10) is divided into two parts that are larger than 0.5 (red) and lower than 0.5 (blue). The error rates in the test and training sets are completely similar which is presented by the red and blue samples distribution. Therefore, there is no indication of over-training [20]. Due to the essence of the linear regression solver, the training and test sets have been performed stably and avoids over training. However, this prevents the model from adapting to the non-linear relationship between variables and objectives. The final optimized operation point is $2.435672,3.364555$, and 0.889564 .

Fig. 11 illustrates transient stress distribution contour of the initial and optimized FE-meshed models in one cycle, in which yield is presented by von Mises stress [8] [12]. The color bars are set to be automatically updated based on the minimum and maximum values of each model. Fig. 11 (a1, b1, c1, and d1) are the initial models at different cycle steps of the transient simulation from $5-100 \%$, respectively. Similarly, the optimized FE models for different transient cycles are 
shown in Fig. 11 (a2, b2, c2, and d2). The maximum stress of $72.22 \mathrm{MPa}$ (occurring in $\mathrm{c} 1$ ) has been reduced to $52.73 \mathrm{MPa}$ (in model c2) which means stress reduction of $19.5 \mathrm{MPa}$,
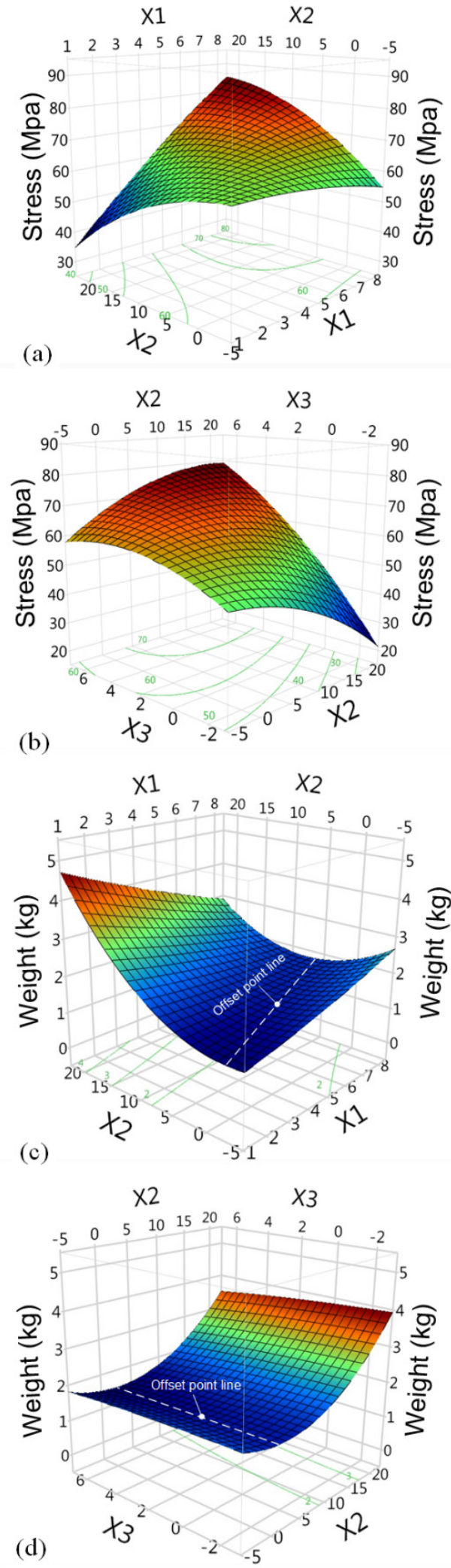

Fig. 9. Response surface of the studied variables on the objectives, where (a) $x_{1}$ and $x_{2}$ variation for $y_{1}$, (b) $x_{2}$ and $x_{3}$ variation for $y_{1}$, (c) $x_{1}$ and $x_{2}$ variation for $y_{2}$, and (d) $x_{2}$ and $x_{3}$ variation for $y_{2}$.

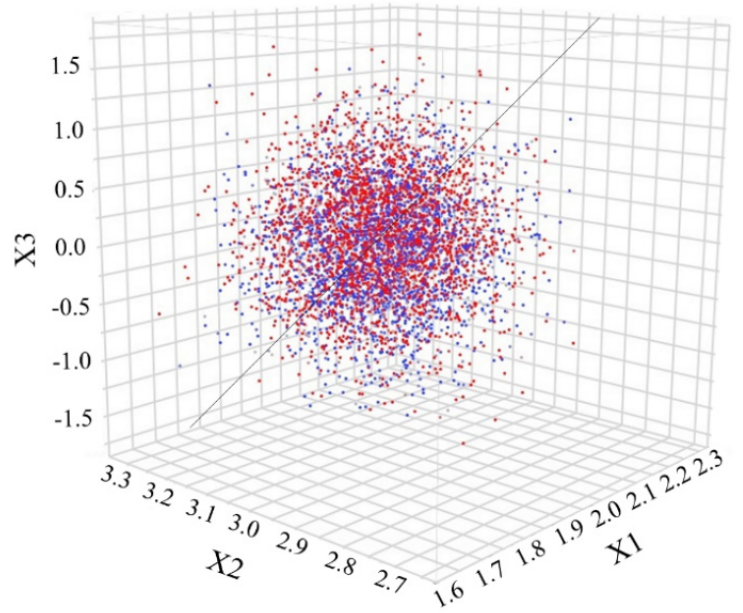

Fig. 10. Generated samples of design operation points around the optimal point using Monte Carlo algorithm based on training and test data sets.

where simultaneously weight reduction from 2.12 to 1.54 could be seen. It should be noted that the electromagnetic forces are calculated based on purely sinusoidal currents meaning that the inverter harmonics are ignored.

The displacement has to be considered carefully when weight reduction is considered as a second objective of the optimization. This is because through a lighter and smaller rotor core, which means lesser body mass particles (rotor core), generally a bigger mechanical stress is predicted. In electrical machine design, displacement results from a stress field induced via normal, tangential and centrifugal forces. The displacement of the rotor core has two components, a rigid-body displacement and a deformation. Deformation matching $(F)$ is one the optimization constraints (as shown in Fig. 6) which means designed model ensures a reliable performance under real operating environments. To verify this challenge of the optimization process, Fig. 12(a) and (b) have presented the deformation of the initial and optimized FE models respectively. The maximum deformation of the optimized model is slightly smaller than the initial model with peak value of $1.003\left(10^{-1} \mathrm{~mm}\right)$. Thus, the optimization constraint is satisfied due to an insignificant change in this parameter. The faded model indicates the model without deformation (at $n=0$ ).

Fig. 13 illustrates how the result of the proposed multilevel-objective methodology affects the rotor in terms of dimensions. The initial model shown in Fig, 13(a) provides maximum stress of $72.22 \mathrm{MPa}$, which can be decreased by 19.5 MPa, using the model presented in Fig. 13(b). Table IV presents the main technical design parameters and $19.5 \mathrm{MPa}$, using the model presented in Fig. 13(b). Table IV presents the main technical design parameters and achievements between both of the three-phase initial and optimized models. Both models are studied under similar electromagnetic conditions such as dimensions, rated speed, achievements between both of the three-phase initial and optimized models. Moreover, the electromagnetic performance of the machine remained constant during the optimization process. It is worthy to mention that both objectives, mechanical stress and weight of rotor core are decreased by $20 \mathrm{MPa}$ and $580 \mathrm{~g}$ of steel sheet, respectively. 

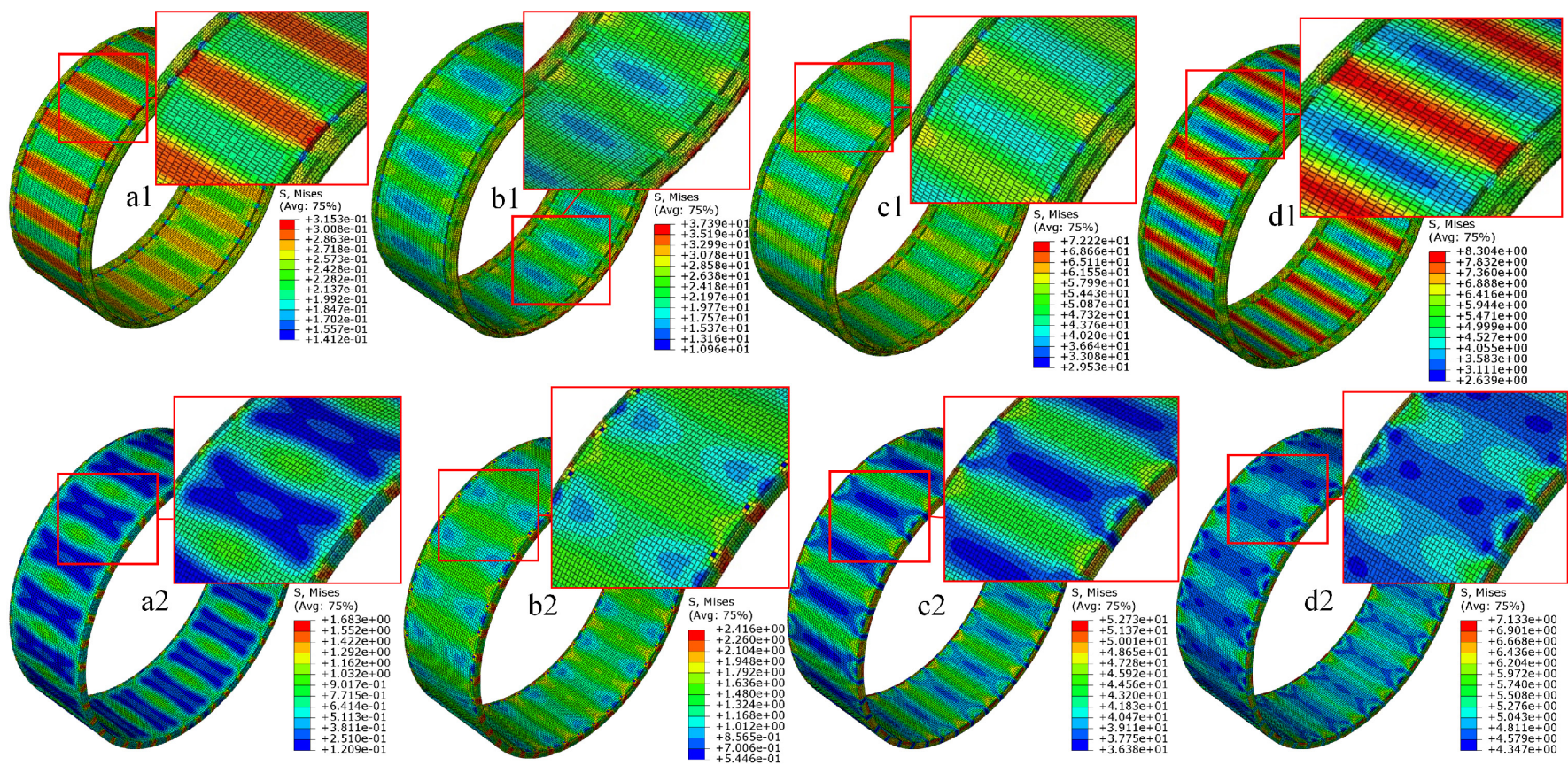

Fig. 11 3-D transient stress computation of the studied models for a period (full cycle rotation), where index 1 and 2 indicate the initial and optimized FE models, (a1) initial @1/4 of transient cycle, (a2) optimized 1/4 of transient cycle, (b1) initial 2/4 of transient cycle, (b2) optimized 2/4 of transient cycle, (c1) initial 3/4 of transient cycle, (c2) optimized 3/4 of transient cycle, (d1) initial 4/4 of transient cycle, and (d2) optimized 4/4 of transient cycle.

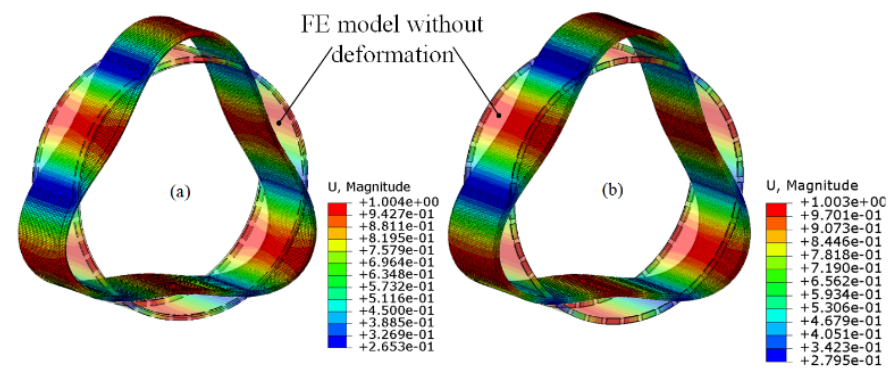

Fig. 12. Deformation and displacement matching of, (a) initial model and (b) optimized model.

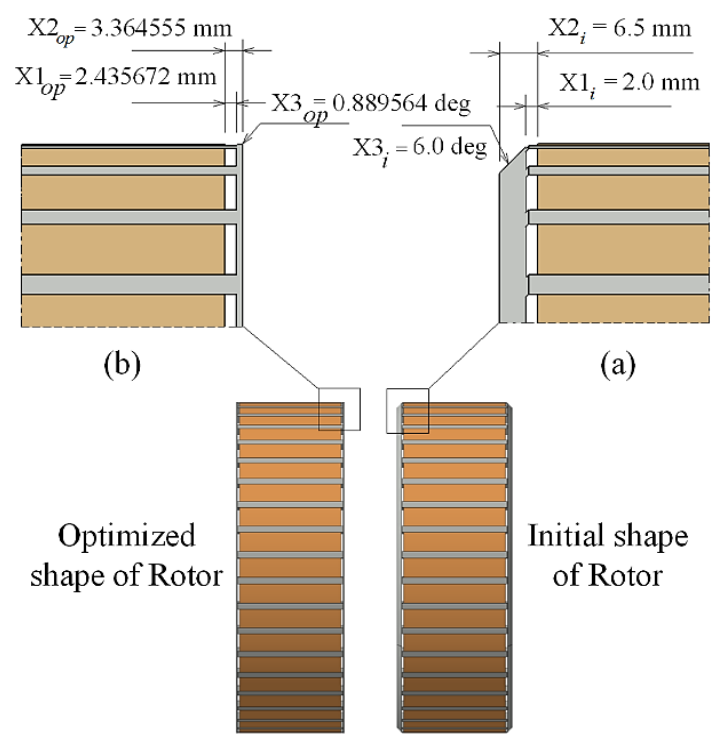

Fig. 13. Geometry of the initial (a) and optimized models (b) with dimensions.

Additionally, the airgap length of the rotor core has been increased by $0.2 \mathrm{~mm}$ in order to avoid any friction between rotor and stator cores based on the maximum displacement (as shown in Fig. 12).
TABLE IV. DESIGN ACHIEVEMENTS BASEd ON SPECIFICATION

\begin{tabular}{|l|c|c|c|}
\hline \multicolumn{1}{|c|}{ Parameters } & Unit & Initial & Optimized \\
\hline$R_{\text {sol }} / R_{\text {si2 }}$ (outer/ inner radius) & $\mathrm{mm}$ & $230 / 115$ & $230 / 115$ \\
\hline Outer/ Inner winding diameters & $\mathrm{mm}$ & $1.8 / 1.2$ & $1.8 / 1.2$ \\
\hline Stack length & $\mathrm{mm}$ & 100 & 100 \\
\hline Rated speed & $\mathrm{rpm}$ & 1000 & 1000 \\
\hline Armature total current density & $\mathrm{A} / \mathrm{mm}^{2}$ & 40 & 40 \\
\hline Airgap & $\mathrm{mm}$ & 1.0 & 1.2 \\
\hline Number of stator slots per core & & 36 & 36 \\
\hline Ratio of pole-arc to pole-pitch & & $0.55 / 4$ & $0.55 / 4$ \\
\hline Outer/ Inner winding turns & & $60 / 28$ & $60 / 28$ \\
\hline Number of poles & & 40 & 40 \\
\hline Rotor core mass & $\mathrm{kg}$ & 2.12 & 1.54 \\
\hline Rated current & $\mathrm{A}$ & 15 & 15 \\
\hline Output electromagnetic torque & $\mathrm{N} . \mathrm{m}$ & 145 & 145 \\
\hline Maximum output power & $\mathrm{kW}$ & 10 & 10 \\
\hline Average cogging torque & $\mathrm{N} . \mathrm{m}$ & 0.854 & 0.994 \\
\hline Maximum stress & $\mathrm{MPa}$ & 72.22 & 52.73 \\
\hline Rotor dynamic eccentricity & $\mathrm{mm}$ & 0.6032 & 0.6032 \\
\hline
\end{tabular}

\section{CONCLUSION}

In this research, a new multi-level-objective optimization methodology has been studied, in which highly-engineered tools were used to minimize both mechanical stress, with a fatigue limitation consideration, and weight of the rotor core of DS-PMBL. All the primary data to fulfill the UP-CCD matrix were collected via a 3-D FEA. Then, a number of fitting models were evaluated to avoid any over fitting and to minimize the error. The model selection is discussed based on $\mathrm{AICc}$ and BIC approaches, which are known as the most two advanced methods in this field. Hence, the 6-terms model was chosen because of its minimized fitting error and sufficient touchback to stay far from the over fitting problem. A response surface method has been used to determine the firstlevel optimal point; afterwards, the second-level optimal point has been found using a Monte Carlo algorithm using training and testing data sets. The optimization has shown that the 
mechanical stress and weight have been decreased significantly by $19.5 \mathrm{MPa}$ and $580 \mathrm{~g}$, respectively. It should be mentioned that the weight (importance) of the desirability function has been set $1(\max )$ and 0.5 for the first and second objectives. The contribution of this research can be significantly used by engineers in optimization studies, regardless of the type of optimization method of which they applied for the first-level achievements.

\section{REFERENCES}

[1] C. Feng, X. Jing, G. Bin, C. Shukang, and Z. Jiange "Double-Stator Permanent Magnet Synchronous in-Wheel Motor for Hybrid Electric Drive System," IEEE Trans. Magnetics, V.45, N.1, pp.278-281, 2009.

[2] S. Niu, K. T. Chau, J. Z. Jiang, and C. Liu "Design and Control of a New Double-Stator Cup-Rotor Permanent-Magnet Machine for Wind Power Generation," IEEE Trans. Magnetics, V. 43, N. 6 pp.2501-2503, 2007.

[3] W-H. Tai, M-C. Tsai, Z-L. Gaing, P-W. Huang and Y-S. Hsu, "Novel Stator Design of Double Salient Permanent Magnet Motor," IEEE Trans. Magnetics, V. 50, N. 4 pp.1-4, 2014.

[4] Y. Wang, M. Cheng, M. Chen, Y. Du, and K. T. Chau, "Design of hightorque-density double-stator permanent magnet brushless motors," IET Electric Power App., V. 5, N. 3, pp.317-323, 2011.

[5] K. Yamazaki, and H. Takeuchi, "Impact of Mechanical Stress on Characteristics of Interior Permanent Magnet Synchronous Motors," IEEE Trans. Industry App., V.53, N.2, pp.963-970, 2017.

[6] J. H. Ahn, J. Y. Choi, C. H. Park, C. Han, C. W. Kim, and T. G. Yoon, "Correlation Between Rotor Vibration and Mechanical Stress in UltraHigh-Speed Permanent Magnet Synchronous Motors," IEEE Trans. Magnetics, V. 53, N. 11, pp. 1-6, 2017.

[7] X. Sun, Z. Xue, J. Zhu, Y. Guo, Z. Yang, L. Chen, and J. Chen "Suspension Force Modeling for a Bearingless Permanent Magnet Synchronous Motor Using Maxwell Stress Tensor Method," IEEE Trans. Applied Superconductivity, V. 26, N. 7, pp.1-5, 2016.

[8] F. Chai, Y. Li, P. Liang, and Y. Pei, "Calculation of the Maximum Mechanical Stress on the Rotor of Interior Permanent-Magnet Synchronous Motors," IEEE Trans. Industrial Electronics, V. 63, N. 6, pp.3420-3431, 2016.

[9] A. H. Isfahani, and B. Fahimi, "Comparison of Mechanical Vibration Between a Double-Stator Switched Reluctance Machine and a Conventional Switched Reluctance Machine," IEEE Trans. Magnetics., V.50, N.2, pp.1-4, 2014.

[10] M. Asgar, and E. Afjei, "Radial Force Reduction in a New Flat-Type Double-Stator Switched Reluctance Motor," IEEE Trans. Energy Conversion, V. 31, N. 1, pp. 141-149, 2016.

[11] W. Wang, M. Luo, E. Cosoroaba, B. Fahimi, and M. Kiani "Rotor Shape Investigation and Optimization of Double Stator Switched Reluctance Machine," IEEE Trans. Magnetics, V. 51, N.3, pp. 1-4, 2015.

[12] K. Yamazaki, and A. Aoki "3-D Electromagnetic Field Analysis Combined With Mechanical Stress Analysis for Interior Permanent Magnet Synchronous Motors," IEEE Trans. Magnetics, V. 52, N.3, pp. $1-4,2016$.

[13] J. W. Jung, B. H. Lee, D. J. Kim, J. P. Hong, J. Y. Kim, S. M. Jeon, and D. H. Songand A. Aoki "Mechanical Stress Reduction of Rotor Core of Interior Permanent Magnet Synchronous Motor," IEEE Trans. Magnetics, V. 48, N. 2, pp. 911-914, 2012.

[14] A. J. P. Ortega, and L. Xu "Investigation of Effects of Asymmetries on the Performance of Permanent Magnet Synchronous Machines," IEEE Trans. Energy Conversion, V. 32, N. 3, pp. 1002-1011, 2017.

[15] K. J. Bathe, Finite Element Procedures. Englewood Cliffs: PrenticeHall, 1996.

[16] P. Asef, R. Bargallo, M. R. Barzegaran, A. C. Lapthorn, and D. Mewes, "Multi-objective Design Optimization Using Dual-Level Response Surface Methodology and Booth's Algorithm for Permanent Magnet Synchronous Generators," IEEE Trans. Energy Conversion, V. PP, N. 99, DOI: 10.1109/TEC.2017.2777397, pp. 1-8, 2017.

[17] H. Akaike, "Akaike's Information Criterion," In: Lovric M. (eds) International Encyclopedia of Statistical Science. Springer, Berlin, Heidelberg, DOI: https://doi.org/10.1007/978-3-642-04898-2, 2011.
[18] Y. Tamura, T. Sato, M. Ooe, and M. Ishiguro, "A procedure for tidal analysis with a Bayesian information criterion," Wiley, V. 104, N. 3, pp. 507-516, DOI: 10.1111/j.1365-246X.1991.tb05697.x, 1991.

[19] J. Ding, V. Tarokh, and Y. Yang, "Bridging AIC and BIC: A New Criterion for Autoregression," IEEE Trans. Information Theory, DOI: 10.1109/TIT.2017.2717599, V. PP, N. 99, pp. 1-26, 2017.

[20] R. A. Irizarry, and M. I. Love, "Data Analysis for the life Science," Lean Publishing process, Chapter book, ISBN-13: 978-1498775670, 2015.

[21] Suresh, S. "Fatigue of Materials," Cambridge University Press. ISBN 0521-57046-8, 2004.

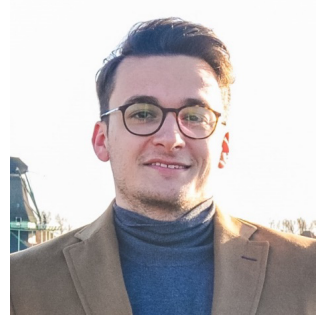

Pedram Asef (M'18) received a B.Sc (Hons.) in Electrical Engineering from Shiraz University, Iran, in 2012, the M.Sc degree from Huazhong University of Science and Technology, China, in 2015, and his Ph.D. degree in Electrical Engineering from the Universitat Politècnica de Catalunya BarcelonaTech (UPC), Barcelona, Spain, in 2018. He won the Chinese Presidential award and Shanghai Jiao Tong University fellowship in 2013 and 2015, respectively. He was also employed by the State Key Laboratory of Advanced Electromagnetic Engineering and Technology (AEET) in China. His previous projects were granted by Texas State Center for Port Management (USA). Currently, he is working for Energy Processing and Integrated Circuits Group (EPIC) at UPC. His fields of interest include electrical machinery and machine drives, renewable energy systems, optimization methods, power systems, electromagnetics, and machine learning.

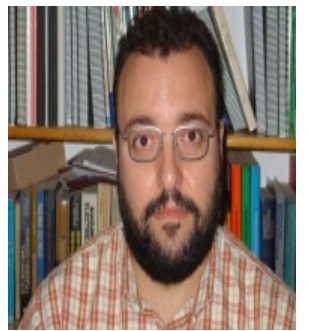

Ramon Bargalló Perpiñà was born in Catalonia, Spain, 1962. He received the B.Sc., M.Sc, and Ph.D. degrees in Industrial Engineering from the Polytechnic University of Catalonia (UPC), in 1985, 1995, and 2001 respectively. $\mathrm{He}$ is currently Professor in Electrical Engineering Department at EEBE of the UPC. Also, he has about +50 papers on International Conferences and Journals. His employment experience includes assessment of some companies about the improvement of design of electrical machines using FE methodology. His research interest is in the field of design of electrical machines.

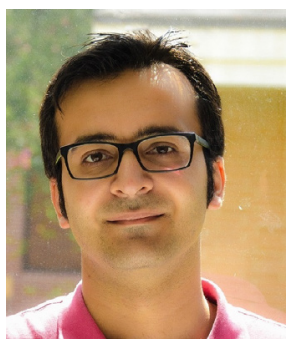

Saeed Moazami received his B.S. degree in Mechanical Engineering from Isfahan University of Technology, Isfahan, Iran, in 2008. He is currently a graduate research assistant at Lamar University, Beaumont, TX, USA, where he is pursuing the Doctoral degree with the Electrical Engineering Department. He is also pursuing a second M.S. degree in Computer Science with Lamar University. Prior to joining Lamar University, he was the head of mechanical engineering department with Behine Faraz Kian company, Isfahan, Iran, from 2012 to 2015. His current research interests include Advanced Robotic Systems, Dynamics, Multi-Agent Systems and Machine Design.

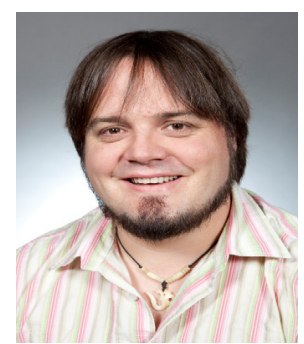

Andrew Lapthorn (M'16) Andrew Lapthorn obtained his NZCE from CPIT in 1999 and his BE (hons) and Ph.D. from the University of Canterbury in 2007 and 2012 respectively. He is now a Lecturer in Electric Machines and High Voltage for the Electrical and Computer Engineering Department of University of Canterbury where he operates the high voltage laboratory. Andrew is involved in a number of research areas including rotating machine and transformer design, high voltage testing of insulation systems, renewable energy systems, engineering education and high temperature superconductivity. 